\title{
[1] Protein Misfolding Cyclic Amplification for Diagnosis and Prion Propagation Studies
}

\author{
By Joaquín Castilla, Paula SaÁ, Rodrigo Morales, Karim Abid, \\ Kinsey Maundrell, and Claudio Soto
}

\begin{abstract}
Diverse human disorders are thought to arise from the misfolding and aggregation of an underlying protein. Among them, prion diseases are some of the most intriguing disorders that can be transmitted by an unprecedented infectious agent, termed prion, composed mainly (if not exclusively) of the misfolded prion protein. The hallmark event in the disease is the conversion of the native prion protein into the disease-associated misfolded protein. We have recently described a novel technology to mimic the prion conversion process in vitro. This procedure, named protein misfolding cyclic amplification (PMCA), conceptually analogous to DNA amplification by polymerase chain reaction (PCR), has important applications for research and diagnosis. In this chapter we describe the rational behind PMCA and some of the many potential applications of this novel technology. We also describe in detail the technical and methodological aspects of PMCA, as well as its application in automatic and serial modes that have been developed with a view to improving disease diagnosis.
\end{abstract}

\section{Introduction}

Prion diseases or transmissible spongiform encephalopathies (TSEs) are neurodegenerative disorders of humans and animals usually characterized by the presence of $\operatorname{PrP}^{\mathrm{res}}$, an abnormal, protease-resistant isoform of a cellular protein called $\operatorname{PrP}^{\mathrm{C}}$. Historically, scrapie has been the most common TSE in animals, affecting sheep for more than 200 years (Collinge, 2001). TSEs have also been identified in mink and mule deer since the 1960s. The most recent and worrisome outbreak of an animal TSE disease is bovine spongiform encephalopathy (BSE) in cattle, which originated in Britain in the 1980s (Prusiner, 1997). BSE has important implications for human health, because the infectious agent can be transmitted to humans producing a new disease, termed variant Creutzfeldt-Jakob disease (vCJD) (Collinge, 1999; Will et al., 1996). TSEs are characterized by an extremely long incubation period, followed by a brief and invariably fatal clinical disease (Roos et al., 1973). To date, no therapy or early diagnosis is available. 
The pathogen responsible for TSEs, called "prion" (Prusiner, 1982), is composed mainly of a misfolded protein named $\mathrm{PrP}^{\mathrm{Sc}}$, which is a posttranslationally modified version of the normal protein, $\operatorname{PrP}^{\mathrm{C}}$ (Cohen and Prusiner, 1998). The conversion seems to involve a conformational change during which the $\alpha$-helical content of the normal protein diminishes and the amount of $\beta$-sheet increases (Caughey et al., 1991; Pan et al., 1993). The structural changes are accompanied by alterations in the biochemical properties: $\operatorname{PrP}^{\mathrm{C}}$ is soluble in nondenaturing detergents, $\operatorname{PrP}^{\mathrm{Sc}}$ is insoluble; $\operatorname{PrP}^{\mathrm{C}}$ is readily digested by proteases, whereas $\operatorname{PrP}^{\mathrm{Sc}}$ is partially resistant, resulting in the formation of a $\mathrm{N}$-terminally truncated fragment known as $\mathrm{PrP}^{\text {res }}$ (Baldwin et al., 1995; Cohen and Prusiner, 1998).

At present, there is no accurate diagnosis for TSEs (Budka et al., 1995; Weber et al., 1997). In the case of sporadic CJD (sCJD) or vCJD, diagnosis is currently based almost entirely on clinical observation, because even though different molecules, such as protein S-100 or the 14-3-3 protein, have been proposed as markers of the disease, none of them are pathognomonic of the syndrome. For this reason, according to the operational diagnosis currently in use by the European Surveillance of CJD, definitive diagnosis can only be established by postmortem neuropathological examination and detection of $\mathrm{PrP}^{\mathrm{res}}$ by immunohistochemistry, histoblot, or Western blot (Budka et al., 1995; Weber et al., 1997). Presymptomatic detection of sCJD or vCJD in living people is currently not possible.

To minimize the propagation of the bovine disease, several tests have been developed to diagnose BSE in postmortem brain tissue (Moynagh and Schimmer, 1999; Soto, 2004). However, in cattle, as in humans, there is no reliable way to identify affected animals early after infection (Schiermeier, 2001), because the problem of a diagnosis on the basis of $\operatorname{PrP}^{\mathrm{res}}$ detection is that this form of the protein is abundant only in the brain at advanced stages of the disease.

Infectivity studies have been used to show that prions are also present in low amounts in peripheral tissues, such as lymphoid organs and blood (Aguzzi, 2000; Brown et al., 2001; Collinge, 2001; Wadsworth et al., 2001), and on the basis of these observations, different bioassays showing high sensitivity have been developed. In these methods, animals are injected with very low quantities of $\mathrm{PrP}^{\mathrm{Sc}}$, and the clinical signs indicating the presence of infectious material are monitored (Brown et al., 2001). The biggest practical problem for using the infectivity assay in routine diagnosis, however, is that prion replication during the incubation phase progresses very slowly, and several months or even years may elapse before a detectable quantity of $\operatorname{PrP}^{\mathrm{Sc}}$ has accumulated in the brain.

In vivo, prion replication is an extraordinary phenomenon that still remains not entirely understood. Although it is known that conversion of 
$\operatorname{PrP}^{\mathrm{C}}$ to $\mathrm{PrP}^{\mathrm{Sc}}$ is an essential element in the etiology of the disease, the intrinsic mechanism by which this occurs, and whether other factors are involved, are crucial questions that remain to be answered.

\section{The Birth of PMCA}

To understand the mechanism of prion conversion, the nature of the infectious agent, and to attempt sensitive diagnosis, we have recently developed a technique referred to as protein misfolding cyclic amplification (PMCA) in which it is possible to simulate prion replication in the test tube in an accelerated mode (Saborio et al., 2001). PMCA is a cyclic process leading to accelerated prion replication (Saborio et al., 2001; Soto et al., 2002). Each cycle is composed of two phases (Fig. 1). During the first phase, the sample, containing minute amounts of $\mathrm{PrP}^{\mathrm{Sc}}$ and a large excess of $\operatorname{PrP}^{\mathrm{C}}$, is incubated to induce formation of $\mathrm{PrP}^{\mathrm{Sc}}$ polymers. In the second phase, the sample is sonicated to break down the polymers, thus multiplying the number of growth sites for subsequent conversion. With each successive cycle, there is an exponential increase in the number of "seeds,"

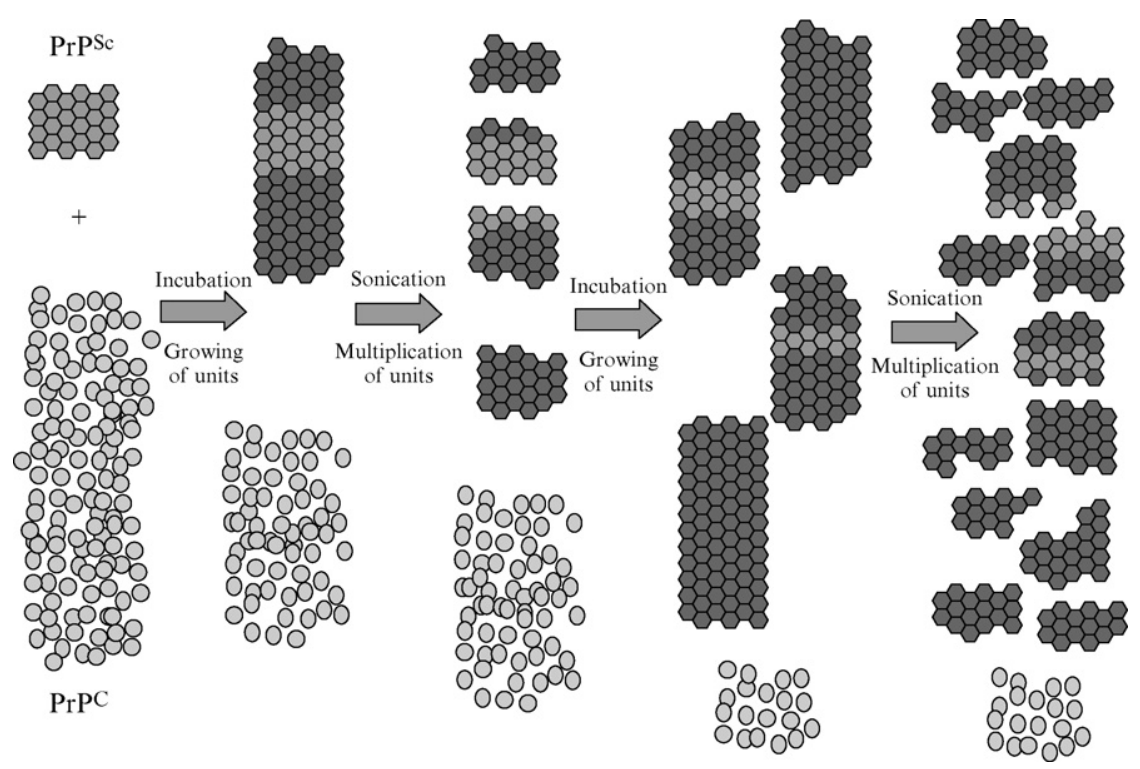

FIG. 1. Diagrammatic representation of principle behind PMCA. Cyclic amplification consists of subjecting a sample containing minute quantities of $\operatorname{PrP}^{\mathrm{Sc}}$ and a large excess of $\mathrm{PrP}^{\mathrm{C}}$ to cycles consisting of phases of growing of polymers and multiplication of converting units. 
and thus the conversion process is dramatically accelerated (Fig. 1) (Saborio et al., 2001). The cyclic nature of the system permits the use of as many cycles as is required to reach the amplification state needed for the detection of $\mathrm{PrP}^{\mathrm{Sc}}$ in a given sample. Recently, we have shown that the in vitro-generated forms of $\mathrm{PrP}^{\mathrm{res}}$ share similar biochemical and structural properties compared with $\mathrm{PrP}^{\mathrm{res}}$ derived from sick brains (Castilla et al., 2005a). Furthermore, inoculation of wild-type hamsters with in vitro-amplified $\mathrm{PrP}^{\text {res }}$ led to a scrapie-like disease identical to the illness produced using infectious material from diseased brains (Castilla et al., 2005a). The technology has been automated, leading to a dramatic increase in efficiency of amplification and its application to detect $\mathrm{PrP}^{\mathrm{Sc}}$ in blood of hamsters experimentally infected with scrapie (Castilla et al., 2005b).

\section{Applications of PMCA}

Simulation of the process of prion conversion using PMCA represents a novel platform technology, which is likely to have a sustained impact in the field of prion biology. By using PMCA, we have been able to demonstrate definitively that the in vitro-generated $\mathrm{PrP}^{\mathrm{res}}$ is fully infectious when injected into wild-type animals (Castilla et al., 2005a). This provides the crucial demonstration that the in vitro conversion that occurs during PMCA closely mimics the events that take place over a protracted period in vivo, leading to disease and, ultimately, to death of the organism. The ability to simulate this process in accelerated mode, under controlled conditions in vitro, thus provides an opportunity to examine many aspects of prion biology that hitherto have been inaccessible to experimentation. Following is a brief description of the multiple areas in which PMCA may contribute.

\section{The Molecular Mechanism of Species Barrier and \\ Prion Strains Phenomena}

As a consequence of the transmission of BSE to humans, a great concern has arisen regarding interspecies infectivity and tissues having a high enough quantity of prions to transmit the disease (Hill et al., 2000; Wadsworth et al., 2001). The molecular aspects that underlie the species barrier and the strain phenomena are still not understood (Bruce, 2003; Clarke et al., 2001; Kascsak et al., 1991). It has been shown that the sequence identity between infectious $\mathrm{PrP}^{\mathrm{Sc}}$ and host prion protein plays a crucial role in determining species barrier (Telling et al., 1996). It is clear that a few amino acid differences between both proteins can modify dramatically the incubation time and the course of the disease (Asante and 
Collinge, 2001; DeArmond and Prusiner, 1996). So far, the investigation of the species barrier, prion strains, and the tissues carrying infectivity has been done mostly using the biological assay of infectivity (Clarke et al., 2001; Wadsworth et al., 2001). However, these studies are time consuming, because it is necessary to wait for several months or even years until the animals develop the clinical symptoms. In addition, the assessment of the species barrier for transmission of prions to humans is compromised by the use of animal models. PMCA can provide a complement to the in vivo studies of the species barrier and prion strains phenomenon by combining $\mathrm{PrP}^{\mathrm{Sc}}$ and $\mathrm{PrP}^{\mathrm{C}}$ from different sources in distinct quantities and evaluating quantitatively the efficiency of the conversion. In this sense, it has to be noted that the cell-free conversion system developed by Caughey and colleagues (Kocisko et al., 1994) has been used successfully to compare and predict species barrier effects and the pertinent underlying mechanisms (Kocisko et al., 1995; Raymond et al., 1997).

\section{Investigation of Factors Involved in the $\operatorname{PrP}^{C}$ to $\operatorname{PrP}^{S c}$ Conversion}

Another important issue in prion propagation is to know whether other factors have any role in the $\operatorname{PrP}^{\mathrm{C}}$ to $\operatorname{PrP}^{\mathrm{Sc}}$ conversion. We reported previously that the conversion procedure does not occur using highly purified prion proteins $\left(\mathrm{PrP}^{\mathrm{Sc}}\right.$ and $\left.\mathrm{PrP}^{\mathrm{C}}\right)$ under our experimental conditions (Saborio et al., 1999). However, the activity is recovered when the bulk of cellular proteins is reincorporated into the sample (Saborio et al., 1999). This finding provides direct evidence that other factors present in the brain are essential to catalyze prion propagation. In this direction, PMCA could also contribute to a better understanding of the mechanism of prion conversion and the identification of additional factors involved. Indeed, Supattapone and coworkers have used PMCA to show that metal cations, such as copper and zinc, and polyanions including diverse types of RNA molecules can modulate PrP conversion in vitro (Deleaut et al., 2003, 2005; Nishina et al., 2004).

\section{Screening for Inhibitors of Prion Propagation}

In the same manner that prion propagation can be used to discover novel drug targets for TSEs in culture cells, PMCA also shows a great advantage in these types of studies. Inhibitors and promoters could be tested quickly in different contexts using human and bovine prions, for which no prion-permissive culture cells have been generated. One of the best targets for TSE therapy is the inhibition and reversal of $\operatorname{PrP}^{\mathrm{C}}$ to $\operatorname{PrP}^{\mathrm{res}}$ conversion (Head and Ironside, 2000; Soto and Saborio, 2001). In drug development, it is crucial to have a relevant and robust in vitro assay to 
screen compounds for activity before testing them in more time-consuming and expensive in vivo assays. PMCA represents a convenient biochemical tool to identify and evaluate the activity of drug candidates for TSE treatment, because it mimics in vitro the central pathogenic process of the disease. Also the simplicity of the method and the relatively rapid outcome are important features of these types of studies. Moreover, the fact that PMCA can be applied to prion conversion in different species provides the opportunity to validate the use in humans of drugs that have been evaluated in experimental animal models of the disease.

\section{Diagnosis}

One of the most valuable applications of PMCA is in TSE diagnosis. As stated previously, the biggest problem facing a biochemical test to detect $\mathrm{PrP}^{\mathrm{res}}$ presymptomatically in tissues other than brain is the very low amount of $\mathrm{PrP}^{\text {res }}$ existing in them. Most of the efforts to develop a diagnostic system for prion diseases have been focused on the increase of sensitivity of the current detection methods. PMCA offers the opportunity to enhance existing methods by amplifying the amount of $\mathrm{PrP}^{\mathrm{res}}$ in the sample. Combining the strategy of reproducing prions in vitro with any of the high-sensitive detection methods, the early diagnosis of TSE may be achieved. The aim would be not only to detect prions in the brain in early presymptomatic cases but also to generate a test to diagnose living animals and humans. For this purpose, a tissue other than brain is required and, to have an easier noninvasive method, detection of prions in body fluids such as urine or blood are the best options. A blood test for CJD can have many applications, including screening of blood banks, identification of populations at risk, reduction of iatrogenic transmission of CJD, and early diagnosis of the disease (Soto, 2004).

\section{Extension to Other Protein Misfolding Diseases}

Besides TSEs, several other diseases involve changes in the conformation of a natural protein to an altered structure with toxic properties capable of inducing tissue damage and organ dysfunction (Carrell and Lomas, 1997; Dobson, 2004; Kelly, 1998; Soto, 2001). This group of diseases called protein misfolding disorders includes several forms of neurodegenerative diseases such as Alzheimer's, Parkinson's, and Huntington's diseases, as well as a group of more than 15 distinct disorders involving amyloid deposition in diverse organs (Soto, 2001). In a similar way to PrPres in TSE, the protein conformational changes associated with the pathogenesis of these diseases result in the formation of abnormal proteins rich in $\beta$-sheet structure, partially resistant to proteolysis and with a high tendency to aggregate (Soto, 
2001). The process of misfolding and aggregation also follows a seedingnucleation mechanism, and hence the principles of PMCA might be applied to amplify the abnormal folding of these proteins as well. Therefore, PMCA may have a broader application for research and diagnosis of diseases in which misfolding and aggregation of a protein are hallmark events.

\section{Method and Technical Details}

Protein misfolding cyclic amplification in its original mode was done by manual operation (Saborio et al., 2001), but we have recently developed an automated mode (aPMCA), which has been developed to increase sensitivity, specificity, and throughput. The increased throughput of aPMCA has allowed us to evaluate the importance of numerous variables including temperature, $\mathrm{pH}$, substrate concentration, type, and concentration of the detergents, power and length of sonication, and so on. In addition, the sensitivity has been increased further by the introduction of a new concept involving serial rounds of amplification. This procedure is named serial automated PMCA (saPMCA) and is similar to application of multiple rounds of PCR amplification to reach high sensitivity detection of DNA.

\section{Buffer}

Conversion Buffer. Composition of the conversion buffer (CB) has been established and optimized after exhaustive studies, and we have found that even small changes may dramatically affect the efficiency of the amplification process. Thus, we highly recommend using the following conversion buffer: PBS; $\mathrm{NaCl}, 0.15 \mathrm{M}$; Triton $\mathrm{X}-100,1 \%$; and complete protease inhibitor cocktail $1 \times$ (Roche, cat\#: 1836145). A pH of between 7.0 and 7.3 is necessary to obtain the best results. Low concentrations of SDS may also be included in CB but are not usually necessary. When used, the SDS concentration should be optimized depending on the type of the $\operatorname{PrP}^{\mathrm{Sc}}$ species to be amplified.

\section{Equipment}

Sonicator. In the original PMCA protocol, the proof of concept was established using a manual sonicator using a single microtip (Saborio et al., 2001). However, with the increased need for high throughput and automation, we have implemented a programmable sonicator that uses a 96-well plate format (Misonix, USA, model S3000MP sonicator) and satisfies the principal requirements for PMCA even though the machine was originally designed for other purposes. Improvements to the equipment planned for the near future should lead to a full adaptation to the needs of PMCA. 
Homogenizer. A principal component in the PMCA reaction is the $\operatorname{PrP}^{\mathrm{C}}$ used as substrate. At this point we consider that a normal brain homogenate $(\mathrm{NBH})$ is the best substrate for high-efficiency amplification. For brain homogenization, we recommend using the high-viscosity mixer Eurostar PWR BSC S1 (IKA, USA), whereas for manual homogenization, the Potter homogenizer is a perfectly satisfactory option.

\section{Preparation of Samples for Amplification}

The correct preparation of the inoculum $\left(\mathrm{PrP}^{\mathrm{Sc}}\right.$ used as starting material) and substrate (material used as source of $\operatorname{PrP}^{\mathrm{C}}$ ) samples is critical to achieve a good efficiency of amplification (Fig. 2). To prepare the best samples for PMCA it is important to know several critical parameters concerning the material to be amplified, in particular, (1) the animal species; (2) the type of tissue in which $\mathrm{PrP}^{\mathrm{Sc}}$ is to be detected; (3) an estimation of the amount of $\mathrm{PrP}^{\mathrm{Sc}}$ in the sample to be amplified; (4) the storage conditions of the sample; and (5) possible inhibitors that may interfere with the amplification.

Whenever possible, it is preferable to use a substrate from the same species as the $\mathrm{PrP}^{\mathrm{Sc}}$ to be amplified (see "Preparation of Substrate"). In this way, any potential problems caused by species barrier can be avoided. On the other hand, the use of substrates from different species can be useful, for example, in studies to understand the nature of the species barrier.

Another important parameter is the condition in which the sample to be amplified has been stored. Although $\operatorname{Pr}^{\mathrm{Sc}}$ is resistant to high temperature (Castilla et al., 2005a), treatment at $>100^{\circ}$ can promote the formation of large aggregates in the samples, which interfere with efficient amplification. The use of samples previously denatured using chaotropic agents or ionic detergents at high concentrations is also incompatible with PMCA and should be avoided. Although not many studies have been done using formalin-fixed samples, it is not recommended to use this type of sample for amplification.

Finally, either the samples to be amplified or the substrates prepared for the amplification may contain potential inhibitory molecules such as plasminogen, cations, or other, as yet, unidentified blood compounds that have been found to interfere with PMCA (data not shown). Because it is extremely important to eliminate such molecules, some samples will require pretreatment before the amplification process (see "Pretreatment in Preparation of Samples from Peripheral Sources"). In addition, special precautions need to be taken when using blood, CSF, saliva, milk, urine, or feces (see "Preparation of Samples from Peripheral Sources").

Preparation of Prion-Infected Samples from CNS. Infectious brain material should be homogenized in conversion buffer (CB) at $10 \%(\mathrm{w} / \mathrm{v})$ 
I. Substrate preparation

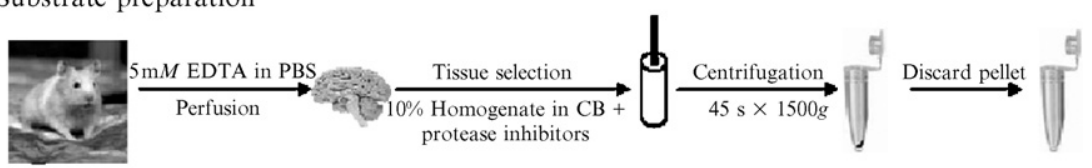

Non infected animal

II. (a) Inoculum preparation (blood)

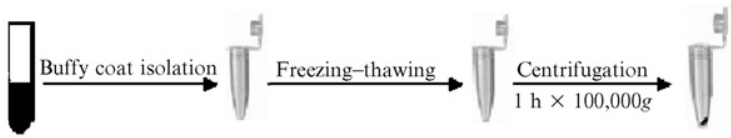

II. (b) Inoculum preparation (other tissues)

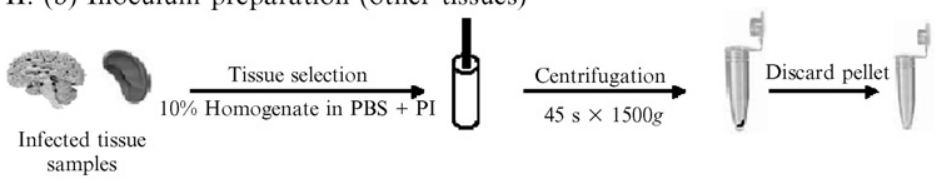

III. Mixing

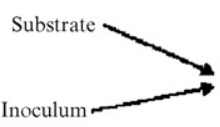

IV. PrPres analysis

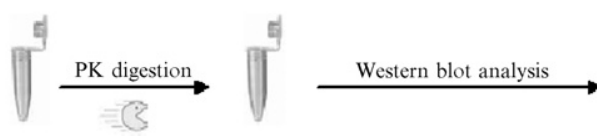

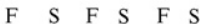
$\underline{1: 1000} \underline{1: 50001: 25,000} \mathrm{C}_{\text {Hа }}$

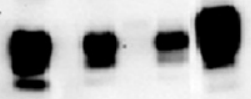

FIG. 2. PMCA method. The scheme shows a diagram of the different procedures involved in PMCA, including the preparation of the substrate (I), inoculum (II), the mixing and amplification process (III), and the $\operatorname{PrP}^{\text {res }}$ detection (IV).

at $4^{\circ}$ using a high-viscosity mixer (see "Equipment"). The homogenate should be centrifuged at $1500 \mathrm{~g}$ for $30 \mathrm{sec}$ and the supernatant retained for further use. Because much of the $\mathrm{PrP}^{\mathrm{Sc}}$ is present in the membrane fractions, centrifugation at a higher speed should be avoided to reduce the risk of losing infectious material in the pellet. Storage of the homogenate in aliquots at $-80^{\circ}$ is recommended.

Preparation of Prion-Infected Samples from Peripheral Sources. Although the most frequent samples for testing will be brain homogenates, other tissues could also be used as a source of infectious material. In most cases, the samples should be prepared in the same manner, with the exception of some special tissues that may require some form of pretreatment. We have 
separated these latter samples into: (1) blood samples, (2) tissue samples containing high amount of blood, and (3) other fluids such as CSF, saliva, milk, urine, and feces.

Blood SAmples. Blood is probably the most interesting sample to use in prion diagnosis. However, it is also considered one of the most complicated tissues for these purposes. The extremely low amount of $\operatorname{PrP}^{\mathrm{Sc}}$ present in blood and the presence of inhibitors of prion replication make it necessary to take special precautions. The blood should be collected using a syringe containing EDTA to avoid clotting and placed in tubes containing sodium citrate. For standard infectious blood material, it is necessary to use at least $1 \mathrm{ml}$ of whole blood. One milliliter of PBS should be added to the total blood and buffy coat should be prepared by centrifugation on a Ficoll gradient using standard procedures. The isolated buffy coat fraction should be subjected to three consecutive freezing-thawing cycles to break the cells, and then centrifuged for $100,000 \mathrm{~g}$ for $1 \mathrm{~h}$ at $4^{\circ}$ to pellet $\mathrm{PrP}^{\mathrm{Sc}}$. For PMCA, the pellet should be resuspended directly in $100 \mu \mathrm{l}$ of normal brain homogenate. The amount of $\mathrm{PrP}^{\mathrm{Sc}}$ present in $1 \mathrm{ml}$ of infectious blood is usually not sufficient for standard aPMCA, and in this case serial rounds of aPMCA are needed (Castilla et al., 2005b) as described later (see "Serial Automatic PMCA Procedure").

Tissue Samples Containing High Amount of Blood. We have observed during development of PMCA that small amounts of plasma or serum can inhibit the PMCA reaction. In addition, the proteinase $\mathrm{K}$ treatment, which is performed after amplification to detect $\mathrm{PrP}^{\mathrm{Sc}}$, can be inhibited by protease inhibitors present in blood. For this reason, whenever possible, it is recommended that animals be perfused with PBS $+5 \mathrm{mM}$ EDTA before dissection of the tissues. When this is not possible, freshly dissected tissues should be washed carefully in PBS $+5 \mathrm{~m} M$ EDTA before the preparation of the samples.

Other Sources of $\mathrm{PRP}^{\mathrm{S}_{\mathrm{C}}}$ such as CSF, Saliva, Milk, Urine, AND FECES. Although we have not yet attempted to use PMCA to amplify samples from these biological fluids, later we provide some advice based on our experience with similar samples. We would recommend diluting these samples with PBS $+5 \mathrm{~m} M$ EDTA followed by centrifugation at $100,000 \mathrm{~g}$ for $1 \mathrm{~h}$. This procedure requires exhaustive washing of the sample with $\mathrm{CB}$ and a further centrifugation at $100,000 \mathrm{~g}$ for $1 \mathrm{~h}$. The pellet, most probably invisible, should be resuspended directly in substrate to avoid more dilution. It is important to note that these special samples could contain enzymes, urea, and other molecules that may coprecipitate with the infectious material, thereby further complicating the amplification step.

Once prepared, samples for amplification should be divided into aliquots and frozen at $-80^{\circ}$. It is known that $\operatorname{PrP}^{\mathrm{Sc}}$ has a strong tendency to 
aggregate, and this seems to increase with repeated freezing and thawing. Although higher levels of aggregation seem not to affect the ability of $\mathrm{PrP}^{\mathrm{Sc}}$ to act as template for amplification (samples have been frozen and retested more than 20 times without any significant difference), the size and number of the large aggregates can lead to errors in sample dilution, which thus may produce variability in the level of amplification.

In vivo studies have shown that the $\mathrm{PK}$-treated $\mathrm{PrP}^{\mathrm{Sc}}$ is still infectious, even though its infectivity is diminished compared with that of nontreated $\mathrm{PrP}^{\mathrm{Sc}}$. The latter may be due to an increased propensity to aggregation. Samples digested with PK treatment can also be amplified by PMCA even though, as with in vivo infectivity, the level of amplification is slightly diminished. Despite this, the use of PK to remove proteinaceous contaminants might be a good option for certain samples.

Preparation of Substrate. We consider the preparation of the substrates to be the most critical step in achieving successful PMCA. In our hands, the best and most convenient substrate is normal brain homogenate from the same species as the prion sample to be amplified, although other substrates have also been used successfully (see "Other Substrates").

Normal Brain Homogenate. As mentioned previously, the presence of cations and certain blood components can seriously affect the amplification process. For this reason, we consider it highly beneficial to perfuse the animals with PBS $+5 \mathrm{~m} M$ EDTA before the brain extraction. After perfusion, a totally white brain can be obtained from the animal. We recommend the use of a $\mathrm{CO}_{2}$ chamber for euthanizing, to avoid using anesthesia, which may also interfere with the subsequent amplification.

Whenever possible, we recommend preparing substrate from animals of the same species as the infectious sample to be amplified. In the case of larger animals such as cattle, sheep, goats, and so on in which perfusion before tissue dissection is not possible, we would recommend removing the entire brain as quickly as possible to reduce postmortem lysis, and then washing the fresh tissue immediately with cold PBS + $5 \mathrm{mM}$ EDTA to remove as much blood as possible. In case obtaining brains from the same species is a problem (e.g., humans), we have successfully used transgenic mice brain overexpressing $\operatorname{PrP}^{\mathrm{C}}$.

We have not experimented extensively at this point to determine which part of the brain is the most suitable for PMCA studies; however, currently we would recommend using the entire encephalic area including brain stem. On the basis of in vivo experiments, we also recommend using animals as young as possible, although we avoid using fetal tissue.

After removal, the brain should be placed into conversion buffer at $4^{\circ}$ and immediately homogenized at $10 \%(\mathrm{w} / \mathrm{v})$ using a high-viscosity mixer (see "Equipment"). In our hands, the highest amplification is obtained using 
$7.5-10 \%$ of substrate, but it is also possible to use $2.5-5 \%$, although with lower yield of amplification. After homogenization, large pieces of tissue and unbroken cells should be removed by a low-speed centrifugation. The low speed $(1500 \mathrm{~g}$ for $30 \mathrm{sec})$ is important to avoid losing or destabilizing membrane components that seem to be essential for conversion. The final substrate preparation should be turbid with visible membrane fragments still present. If the homogenate is transparent, the efficiency of conversion will not be good.

Homogenates, once prepared, should be stored at $-80^{\circ}$ and can be thawed and refrozen approximately 10 times without significant loss of efficiency. Storage at $-20^{\circ}$ is not recommended; however, for short-term use, homogenates can be kept at $4^{\circ}$ for up to 7 days. Following the studies from Supattapone's group (Deleault et al., 2003), it is recommended that the work be done in RNAse-free conditions.

Other Substrates. The normal brain homogenate is considered, at this moment, to be the most efficient substrate for amplification, particularly for TSE diagnosis purposes. However, the use of alternative substrates could be beneficial for other studies aimed, for example, at understanding the tissues capable to propagate prions or to localize other factors involved in $\operatorname{PrP}^{\mathrm{C}}$ to $\operatorname{PrP}^{\mathrm{Sc}}$ conversion.

Cells. Protein extracts from whole cells can provide a good substrate for specific applications of PMCA. For these studies, transient or stably transfected knock out-PrP (PrP-KO) cells overexpressing different $\operatorname{PrP}^{\mathrm{C}}$ transgenes can provide useful substrates for subsequent experimentation. Our experience at this point has been focused on PrP knock out N2a cells overexpressing hamster or mouse $\mathrm{PrP}$ as substrates. Cells should be resuspended in a small volume of PBS $(0.5-1 \mathrm{ml})$ and centrifuged in a $1.5-\mathrm{ml}$ Eppendorf tube for $5 \mathrm{~min}$ at $2000 \mathrm{~g}$. The supernatant must be completely removed, and the pellet containing cells should be resuspended in 50-100 $\mu \mathrm{l}$ of $\mathrm{CB}$ and the centrifugation step repeated. To enhance the level of amplification using cells as substrate, we have found that it is useful to supplement the PMCA reaction with a "PrP inert substrate" such as PrP-KO brain homogenate or normal brain homogenate from a species resistant to prion propagation such as rabbit. This material provides additional quantities of a yet unknown "conversion factor," which is highly expressed in brain.

LIPID RAFTs. The detergent-resistant membrane (DRM) or lipid-raft fraction, should also be considered as a good alternative substrate to the whole brain homogenate. $\operatorname{PrP}^{\mathrm{C}}$ is attached to the outer cell membrane by a glycosyl phosphatidylinositol (GPI) anchor, and, like other GPI-anchored proteins, $\operatorname{Pr}^{\mathrm{C}}$ is found in the cholesterol, glycosphingolipid, sphingomyelinrich membrane subdomains known as lipid rafts (Vey et al., 1996). This 
membrane fraction seems to contain all elements required for prion conversion (unpublished data). Various methods have been described to isolate lipid rafts both from brain homogenates and from neuronal cell lines. We routinely use Optiprep (Axis-Shield) density gradients to isolate lipid raft from N2a neuroblastoma cells or from brain.

PURIFIEd PRP ${ }^{\mathrm{C}}$. Currently, one of the most intriguing issues in the prion field is the identity of the factors required for $\operatorname{PrP}^{\mathrm{C}}$ to $\operatorname{PrP}^{\mathrm{Sc}}$ conversion. With this in mind, we have designed a purification technique to obtain $\operatorname{PrP}^{\mathrm{C}}$ free of other components but suitable for conversion to $\operatorname{PrP}^{\mathrm{Sc}}$. This purified substrate can be used to study the effects of well-characterized biological fractions on the conversion process. Mixing $\operatorname{PrP}^{\mathrm{C}}$ with a sample that is able to complement the conversion enables us to have a better understanding of the requirements of the conversion process (e.g., nucleic acids, lipids, proteins). The ability to purify $\operatorname{PrP}^{\mathrm{C}}$ will allow us to mix it with $\operatorname{PrP}^{\mathrm{Sc}}$ and screen for cellular components capable of reconstituting a conversion competent environment. This is currently being worked out and will be reported at a later time.

\section{Automated PMCA Procedure}

In the original PMCA procedure (Saborio et al., 2001), sonication was performed manually using a single probe sonicator. More recently, an automated version of PMCA has been developed (Castilla et al., 2005a) that shows improved efficiency and reproducibility. This procedure now referred to as automatic PMCA (aPMCA) uses an inverted 96-well sonicator that can be programmed for automatic operation (see "Equipment"). This technique has proved to be of great value for diagnosis and for other prion propagation studies. aPMCA overcomes one of the major drawbacks of manual PMCA, namely cross-contamination, because there is no direct contact between the sonicator probe and the sample. The following recommendations for standard use of this procedure should be observed:

1. Samples to be amplified are placed at different dilutions into $0.2-\mathrm{ml}$ PCR tubes and mixed with $10 \%$ substrate (see "Preparation of Substrate"). The final volume should be between 60 and $100 \mu$ l. For each condition, three tubes are prepared. One is frozen immediately (frozen control), and the second is subjected to multiple cycles of incubation/sonication (PMCA samples) (Fig. 2).

2. Samples are incubated for $30-60 \mathrm{~min}$ at $37^{\circ}$ in the reservoir of the automatic sonicator. The duration of the incubation phase needs to be optimized for each sample, because factors such as the prion strain and the amount of $\operatorname{PrP}^{\mathrm{Sc}}$ in the sample will require different incubation periods. There are numerous parameters that can be modified (including time, 
temperature, and agitation rate) to reach highest efficiency for a particular sample; however, in this chapter we will limit the description to our standard procedure. As further knowledge about prion replication in vitro accumulates, and as more sophisticated equipment becomes available, additional modifications to the technique will be implemented.

3. Samples are sonicated for a single pulse of $40 \mathrm{sec}$. The sonication is the most critical step in this technique, and variation in the level of sonication can generate huge differences in the results. Using the optimal level of sonication is crucial to break down and multiply the $\operatorname{PrP}^{\mathrm{Sc}}$ polymers without affecting their capacity to act as "seed" for further $\operatorname{PrP}^{\mathrm{C}}$ conversion. It is also important to note that the ultrasound strength needed to amplify $\mathrm{PrP}^{\mathrm{Sc}}$ of distinct strains and from diverse species can be different (Soto et al., 2005), and hence low or even no amplification at all may be obtained for new samples under conditions that work very well for others. These findings are probably related to the specific conformation/ aggregation state of each strain of prion, which has been proposed to explain the differences in clinical, pathological, and biochemical features of distinct strains.

The sonication step is the most difficult to monitor adequately, and many factors can influence the final amplification observed. These factors, which we describe later, need to be taken into account to achieve maximal amplification.

a. Power of sonication: The power of sonication for the $263 \mathrm{~K}$ hamster prion strain should be set to the maximum potency of this sonicator (level 8-10). For other species/strains, sonication power should be optimized experimentally and is in general lower than for $263 \mathrm{~K}$ strain.

b. Wavelength: At present, we do not know how wavelength affects the effectiveness of sonication; however, we should be able to determine this once other equipment becomes available.

c. Water in the sonication reservoir: The reservoir has to be filled with $140 \mathrm{ml}$ of water (see "Equipment"), which decreases at a rate of around $2.5 \mathrm{ml} / \mathrm{h}$ at $37^{\circ}$. Tubes should be incubated without touching the sonication plate.

d. Tubes for sonication: It is very important to use thin-walled 0.2-ml tubes to obtain the most effective penetration of ultrasound waves.

e. Number of tubes: The rack used in this sonicator is designed to hold 96 tubes. However, our experiments have shown that the effective power of the sonicator diminishes when the rack is completely full. This is probably because each tube attenuates to some extent the effect of the ultrasound waves. If all positions need to be used, we would recommend increasing the power of sonication. In our 
standard procedure, only $60 \%$ of the rack is used, with tubes being placed at random positions across the plate.

4. The incubation/sonication cycle (steps 2-3) should be repeated as many times as needed to reach the desired level of amplification. For the standard reaction, we recommend approximately $24 \mathrm{~h}$ of cyclic amplification.

It is preferable to complete the entire amplification experiment without freezing-thawing the samples. If it is necessary to interrupt the amplification, samples should always be frozen at $-80^{\circ}$. It is not necessary to use a quick freezing procedure.

Although the theoretical limit of amplification will be the amount of $\operatorname{PrP}^{\mathrm{C}}$ substrate present in the tube, we regularly observe that the efficiency of amplification starts to decrease after approximately 150 cycles $(75 \mathrm{~h}$ of incubation). This problem is most likely the result of a deleterious effect of prolonged incubation at $37^{\circ}$. Under these conditions, the $\operatorname{PrP}^{\mathrm{C}}$ substrate or other brain-derived cofactors necessary to promote the conversion of $\operatorname{PrP}^{\mathrm{C}}$ into $\mathrm{PrP}^{\mathrm{Sc}}$ might be altered or consumed. In view of this, and to increase further the level of achievable amplification, we have extended the technique of aPMCA to include serial rounds, in which at each new round, the amplified samples are rediluted into fresh substrate. This new approach, termed serial automated PMCA (saPMCA), will be described in the following.

To maintain a good reproducibility of the aPMCA technique some points need to be carefully considered, especially when small amounts of $\mathrm{PrP}^{\mathrm{Sc}}$ are used for amplification. In particular, the following situations should be avoided:

a. Low sample volumes $(<50 \mu \mathrm{l})$.

b. Low water level $(<100 \mathrm{ml})$ in the reservoir of the automatic sonicator.

c. Bubble formation in the sample that could prevent a good transmission of sonication waves.

5. After the last pulse of sonication is completed, the samples are ready for protease $\mathrm{K}$ digestion (see "Detection of Amplified Product"). If digestion is not performed immediately, the amplified samples should be stored at $-80^{\circ}$.

\section{Serial Automatic PMCA (saPMCA) Procedure}

Serial automatic PMCA consists of successive rounds of aPMCA in which at each round the amplified sample is diluted into fresh substrate. This approach is highly recommended for experiments requiring elevated levels of amplification, especially when working with samples containing minute initial amounts of $\mathrm{PrP}^{\mathrm{Sc}}$, such as blood, CSF, or peripheral nonlymphoid tissues. 
As mentioned previously, the efficiency of PMCA decreases after approximately $75 \mathrm{~h}$ of constant incubation at $37^{\circ}$. However, efficient conversion is restored when the amplified samples are diluted into fresh substrate. Therefore, after a first round of standard aPMCA, the amplified samples are diluted into NBH and amplified in a second round. The dilution factor depends on the purpose of the study and the original dilution of the $\operatorname{PrP}^{\mathrm{Sc}}$. For experiments in which the aim is to simply eliminate the original inoculum (e.g., for comparative studies, infectivity experiments), and where the initial amount of $\operatorname{PrP}^{\mathrm{Sc}}$ is relatively high $\left(4 \log \mathrm{LD}_{50}\right)$, a $100-$ or 1000 -fold dilution can be performed at each stage of saPMCA. However, in studies where undetectable amounts or $\mathrm{PrP}^{\mathrm{Sc}}$ are present, even after a first round of PMCA, a 10-fold dilution is enough to refresh the substrate. The rounds of saPMCA can be repeated as many times as is needed to reach the detection threshold of Western blotting. Samples remaining negative after eight rounds of saPMCA can be considered negative, because according to our experience, approximately six rounds of saPMCA can amplify the minimum amount of material required for amplification (approximately a few hundred molecules of $\mathrm{PrP}^{\mathrm{Sc}}$ monomers) (unpublished data).

Because of the PCR-like nature of PMCA, special care should be taken during the manipulation of the samples when performing serial dilutions of the amplified material. Thus, after each round of aPMCA, the samples should be gently spun down to remove material present in the lid, which arises during sonication or because of condensation of the sample. Given the power of this procedure, inclusion of negative control samples $(\mathrm{NBH}$ without $\mathrm{PrP}^{\mathrm{Sc}}$ ) that are amplified and serially diluted in parallel with the experimental samples is highly recommended.

For safety conditions, filter tips should be used for liquid handling, and sonication should be performed in a closed container inside a BSL-2 hood to avoid the spread of infectious material.

\section{Detection of Amplified Product}

After amplification, the two samples (amplified and frozen) are digested with proteinase $\mathrm{K}(\mathrm{PK})$, and $\mathrm{PrP}^{\mathrm{res}}$ is detected by immunological methods. Because distinct species/strains of prion show a different extent of resistance to proteolytic degradation, the optimal PK treatment condition should be determined beforehand. The critical issue is to make sure that no $\operatorname{PrP}^{\mathrm{C}}$ remains undigested after $\mathrm{PK}$ treatment, because it is a common mistake to confuse incomplete digestion of $\operatorname{PrP}^{\mathrm{C}}$ with false-positive $\operatorname{PrP}^{\mathrm{res}}$ formation. When PrP is detected by Western blotting, it is easy to distinguish incomplete $\operatorname{PrP}^{\mathrm{C}}$ digestion from bona-fide $\operatorname{PrP}^{\mathrm{res}}$, because the latter exhibit a switch on molecular weight because of the removal of the first $\sim 90$ amino acids. To ensure complete digestion, especially after extended incubations, a higher 
concentration of PK may be required to digest increasingly larger aggregates. Addition of up to $0.05 \%$ SDS in the buffer used for the PK treatment may also help. Digestions using temperatures between $42^{\circ}$ and $64^{\circ}$ and with shaking at $350-450 \mathrm{rpm}$ are also recommended. Our standard procedure, which can be taken as a basis for further optimization, is as follows.

1. Prion-containing samples $(20 \mu \mathrm{l})$ are incubated with standard concentrations of PK $(50 \mu \mathrm{g} / \mathrm{ml}$ for hamster, $25 \mu \mathrm{g} / \mathrm{ml}$ for mouse, $20 \mu \mathrm{g} / \mathrm{ml}$ for cattle, or $40-50 \mu \mathrm{g} / \mathrm{ml}$ for CJD) for $1 \mathrm{~h}$ at $45^{\circ}$ with shaking at $350-450 \mathrm{rpm}$. Aliquots of PK $(10 \mathrm{mg} / \mathrm{ml})$ are stored frozen at $-20^{\circ}$, and in the interests of reproducibility, any thawed, unused enzyme is discarded at the end of the experiment. Note: Blood contains protease inhibitors that can interfere with the PK digestion, and in those samples where the presence of blood is unavoidable, the PK concentration should be adjusted accordingly.

2. The PK digestion is stopped by addition of phenyl-methyl-sulfonylfluoride or SDS-PAGE loading buffer. Samples can be analyzed for the presence of $\mathrm{PrP}^{\mathrm{res}}$ using any of the established immunological methods, such as Western blotting or ELISA (Soto, 2004).

\section{Acknowledgments}

This work is partially supported by NIH grants AG0224642 and NS049173. Dr. Soto is part of the European Community project TSELAB.

\section{References}

Aguzzi, A. (2000). Prion diseases, blood and the immune system: Concerns and reality. Haematologica 85, 3-10.

Asante, E. A., and Collinge, J. (2001). Transgenic studies of the influence of the PrP structure on TSE diseases. Adv. Protein Chem. 57, 273-311.

Baldwin, M. A., Cohen, F. E., and Prusiner, S. B. (1995). Prion protein isoforms, a convergence of biological and structural investigations. J. Biol. Chem. 270, 19197-19200.

Brown, P., Cervenakova, L., and Diringer, H. (2001). Blood infectivity and the prospects for a diagnostic screening test in Creutzfeldt-Jakob disease. J. Lab. Clin. Invest. 137, 5-13.

Bruce, M. E. (2003). TSE strain variation. Br. Med. Bull. 66, 99-108.

Budka, H., Aguzzi, A., Brown, P., Brucher, J. M., Bugiani, O., Gullotta, F., Haltia, M., Hauw, J. J., Ironside, J. W., Jellinger, K., Kretzschmar, H. A., Lantos, P. L., Masullo, C., Schlote, W., Tateishi, J., and Weller, R. O. (1995). Neuropathological diagnostic criteria for Creutzfeldt-Jakob disease (CJD) and other human spongiform encephalopathies (Prion diseases). Brain Pathol. 5, 459-466.

Carrell, R. W., and Lomas, D. A. (1997). Conformational disease. Lancet 350, 134-138.

Castilla, J., Saá, P., Hetz, C., and Soto, C. (2005a). In vitro generation of infectious scrapie prions. Cell 121, 195-206.

Castilla, J., Saá, P., and Soto, C. (2005b). Detection of prions in blood. Nat. Med. 11, 982-985.

Caughey, B. W., Dong, A., Bhat, K. S., Ernst, D., Hayes, S. F., and Caughey, W. S. (1991). Secondary structure analysis of the scrapie-associated protein $\operatorname{PrP} 27-30$ in water by infrared spectroscopy. Biochemistry 30, 7672-7680. 
Clarke, A. R., Jackson, G. S., and Collinge, J. (2001). The molecular biology of prion propagation. Philos. Trans. R. Soc. Lond. B Biol. Sci. 356, 185-195.

Cohen, F. E., and Prusiner, S. B. (1998). Pathologic conformations of prion proteins. Ann. Rev. Biochem. 67, 793-819.

Collinge, J. (1999). Variant Creutzfeldt-Jakob disease. Lancet 354, 317-323.

Collinge, J. (2001). Prion diseases of humans and animals: Their causes and molecular basis. Annu. Rev. Neurosci. 24, 519-550.

DeArmond, S. J., and Prusiner, S. B. (1996). Transgenetics and neuropathology of prion diseases. Curr. Top. Microbiol. Immunol. 207, 125-146.

Deleault, N. R., Lucassen, R. W., and Supattapone, S. (2003). RNA molecules stimulate prion protein conversion. Nature 425, 717-720.

Deleault, N. R., Lucassen, R. W., and Supattapone, S. (2005). PrPres amplification reconstituted with purified prion proteins and synthetic polyanions. J. Biol. Chem. 280, 26873-26879.

Dobson, C. M. (2004). Protein chemistry. In the footsteps of alchemists. Science 304, 1259-1262.

Head, M. W., and Ironside, J. W. (2000). Inhibition of prion protein conversion: A therapeutic tool? Trends Microbiol. 6, 6-8.

Hill, A. F., Joiner, S., Linehan, J., Desbruslais, M., Lantos, P. L., and Collinge, J. (2000). Species-barrier-independent prion replication in apparently resistant species. Proc. Natl. Acad. Sci. USA 97, 10248-10253.

Kascsak, R. J., Rubenstein, R., and Carp, R. I. (1991). Evidence for biological and structural diversity among scrapie strains. Curr. Top. Microbiol. Immunol. 172, 139-152.

Kelly, J. W. (1998). The alternative conformations of amyloidogenic proteins and their multistep assembly pathways. Curr. Opin. Struct. Biol. 8, 101-106.

Kocisko, D. A., Come, J. H., Priola, S. A., Chesebro, B., Raymond, G. J., Lansbury, P. T., and Caughey, B. (1994). Cell-free formation of protease-resistant prion protein. Nature 370, 471-474.

Kocisko, D. A., Priola, S. A., Raymond, G. J., Chesebro, B., Lansbury, P. T., Jr., and Caughey, B. (1995). Species specificity in the cell-free conversion of prion protein to protease-resistant forms: A model for the scrapie species barrier. Proc. Natl. Acad. Sci. USA 92, 3923-3927.

Moynagh, J., and Schimmer, H. (1999). Test for BSE evaluated. Nature 400, 105.

Nishina, K., Jenks, S., and Supattapone, S. (2004). Ionic strength and transition metals control PrPSc protease resistance and conversion-inducing activity. J. Biol. Chem. 43, 2613-2621.

Pan, K. M., Baldwin, M., Njuyen, J., Gassett, M., Serban, A., Groth, D., Mehlhorn, I., and Prusiner, S. B. (1993). Conversion of alpha-helices into b-sheets features in the formation of scrapie prion proteins. Proc. Natl. Acad. Sci. USA 90, 10962-10966.

Prusiner, S. B. (1982). Novel proteinaceous infectious particles cause scrapie. Science 216, 136-144.

Prusiner, S. B. (1997). Prion diseases and the BSE crisis. Science 278, 245-251.

Raymond, G. J., Hope, J., Kocisko, D. A., Priola, S. A., Raymond, L. D., Bossers, A., Ironside, J., Will, R. G., Chen, S. G., Petersen, R. B., Gambetti, P., Rubenstein, R., Smits, M. A., Lansbury, P. T., Jr., and Caughey, B. (1997). Molecular assessment of the potential transmissibilities of BSE and scrapie to humans. Nature 388, 285-288.

Roos, R., Gajdusek, D. C., and Gibbs, C. J., Jr. (1973). The clinical characteristics of transmissible Creutzfeldt-Jakob disease. Brain 96, 1-20.

Saborio, G. P., Permanne, B., and Soto, C. (2001). Sensitive detection of pathological prion protein by cyclic amplification of protein misfolding. Nature 411, 810-813.

Saborio, G. P., Soto, C., Kascsak, R. J., Levy, E., Kascsak, R., Harris, D. A., and Frangione, B. (1999). Cell-lysate conversion of prion protein into its protease-resistant isoform suggests the participation of a cellular chaperone. Biochem. Biophys. Res. Commun. 258, 470-475.

Schiermeier, Q. (2001). Testing times for BSE. Nature 409, 658-659. 
Soto, C. (2001). Protein misfolding and disease; protein refolding and therapy. FEBS Lett. 498, 204-207.

Soto, C. (2004). Diagnosing prion diseases: Needs, challenges and hopes. Nat. Rev. Microbiol. 2, 809-819.

Soto, C., and Saborio, G. P. (2001). Prions: Disease propagation and disease therapy by conformational transmission. Trends Mol. Med. 7, 109-114.

Soto, C., Saborio, G. P., and Anderes, L. (2002). Cyclic amplification of protein misfolding: Application to prion-related disorders and beyond. Trends Neurosci. 25, 390-394.

Soto, C., Anderes, L., Suardi, S., Cardone, F., Castilla, J., Frossard, M. J., Peano, S., Saá, P., Limido, L., Carbonatto, M., Ironside, J., Torres, J. M., Pocchiari, M., and Tagliavini, F. (2005). Pre-symptomatic detection of prions by cyclic amplification of protein misfolding. FEBS Lett. 579, 638-642.

Telling, G. C., Parchi, P., DeArmond, S. J., Cortelli, P., Montagna, P., Gabizon, R., Mastrianni, J., Lugaresi, E., Gambetti, P., and Prusiner, S. B. (1996). Evidence for the conformation of the pathologic isoform of the prion protein enciphering and propagating prion diversity. Science 274, 2079-2082.

Vey, M., Pilkuhn, S., Wille, H., Nixon, R., DeArmond, S. J., Smart, E. J., Anderson, R. G., Taraboulos, A., and Prusiner, S. B. (1996). Subcellular colocalization of the cellular and scrapie prion proteins in caveolae-like membranous domains. Proc. Natl. Acad. Sci. USA 93, 14945-14949.

Wadsworth, J. D., Joiner, S., Hill, A. F., Campbell, T. A., Desbruslais, M., Luthert, P. J., and Collinge, J. (2001). Tissue distribution of protease resistant prion protein in variant Creutzfeldt-Jakob disease using a highly sensitive immunoblotting assay. Lancet 358, 171-180.

Weber, T., Otto, M., Bodemer, M., and Zerr, I. (1997). Diagnosis of Creutzfeldt-Jakob disease and related human spongiform encephalopathies. Biomed. Pharmacother. 51, 381-387.

Will, R. G., Ironside, J. W., Zeidler, M., Cousens, S. N., Estibeiro, K., Alperovitch, A., Poser, S., Pocchiari, M., Hofman, A., and Smith, P. G. (1996). A new variant of Creutzfeldt-Jakob disease in the UK. Lancet 347, 921-925.

\title{
[2] Fractionation of Prion Protein Aggregates by Asymmetrical Flow Field-Flow Fractionation
}

\author{
By Jay R. Silveira, Andrew G. Hughson, and Byron Caughey
}

\begin{abstract}
Achieving the successful separation and analysis of amyloid and other large protein aggregates can be a difficult proposition. Field-flow fractionation (FFF) is a flow-based separation method like chromatography; however, FFF is capable of high-resolution separations in the absence of a stationary matrix. Thus, FFF is a relatively gentle technique and is well suited to the task of separating large macromolecules and macromolecular
\end{abstract}

